\title{
EXPLORING CORRELATION USING CIRCLE WAVES
}

\author{
Dhruv Ishpuniani \\ Trinity College Dublin, The University of Dublin, Ireland
}

\begin{abstract}
Correlation is widely used in data analytics applications to measure association between various variables. The common approaches to visualize multivariate correlation are clustered heatmaps and scatterplot matrices, however, they do not scale well when the number of variables is large. This paper presents a new animated, aesthetically pleasing alternative to the aforementioned approaches which also reduces the information overload experienced by the viewer. The proposed technique is applicable to scenarios where the analysis requires multiple target variables to be investigated and a high-level intuition about the correlation magnitude is enough.
\end{abstract}

\section{KEYWORDS}

Correlation, Exploratory Data Analysis, Multivariate Data, Circle Waves

\section{INTRODUCTION}

Correlation is a method to gauge the strength of association between two discrete or continuous, quantitative variables. Pearson's correlation coefficient is a widely used metric to measure correlation and it captures a magnitude of the linear relationship between features. Pearson's correlation has a wide variety of applications, from selecting features for machine learning models (Blum and Langley, 1997) to finding genes that are related to a specific disease network (Horvath and Dong, 2008). Visualizing correlation helps in exploratory data analysis by providing the investigator with a platform to test new hypotheses.

Idioms such as clustered heatmaps and scatterplot matrices are generally utilized to visualize correlation. Both these approaches are reasonably effective in visualizing correlations; however, they lose their effectiveness when the number of features increases. Additionally, they both suffer from screen space limitations.

The main contribution of this paper is to provide an alternative to heatmaps and scatterplots to visualize correlations in the case where a high-level intuition about the variables highly correlated with the target variable is enough and the number of variables is very large. Additionally, the technique is useful where the analysis requires investigating multiple such target variables. The animated, aesthetically pleasing, novel technique to visualize multivariate correlation presented in this paper, reduces the information overload experienced by the viewer and is able to convey the correlation information better.

\section{BACKGROUND AND RELATED WORK}

A Scatterplot is one of the most basic idioms used to visualize the correlation between two variables (Rensink and Baldridge, 2010). The two axes represent variables and each plotted point indicates the value observed for the two variables. The magnitude of correlation is measured by calculating the deviation from the straight line produced by the point cloud. Scatterplot matrices are used to compare more than two variables. A scatterplot matrix (figure 1(a)) contains scatterplots where each scatterplot has the target variable on one axis and a variable with which the correlation has to be visualized on the other axis. This approach provides a very rich visualization, but at the same time, it also requires a lot of screen-space. Additionally, scatterplot matrices do not scale well to explore more than a few dozen variables in a dataset (Friendly, 2002). 
Another standard approach is to compute correlation coefficients between all variable pairs and visualizing the correlation coefficient matrix using clustered heatmaps (Wilkinson and Friendly, 2009) (figure 1(b)). Colour is used to encode the pairwise correlation information. Clustering helps with detecting patterns; however, the detection of patterns depends on the clustering technique used. Along with that, due to the relative nature of colour perception, it is challenging to accurately interpret the visualization (Wong, 2010).

The original idea for circle waves as a visual was proposed by Dave Whyte (Whyte, 2014). The idea of using circle waves to show correlation between various fields was inspired from the solar correlation map (Zapf and Kraushaar, 2017). This approach visualizes the Pearson's correlation coefficients using the model of a solar system, with the target variable being in place of the sun. The magnitude of correlation is encoded in the radius of orbit, higher the correlation, shorter the radius. The kind of correlation (positive or negative) is encoded by the colour of the label.

Research has suggested that viewers find it easier to interpret visualizations containing animated elements (Robertson et al., 2008). In addition, aesthetics play an important role in engaging the viewer with a particular visualization (Cawthon and Moere, 2007). The technique proposed in this paper builds upon the concepts of motion and aesthetics to effectively visualize correlation while combating the limitations of the previous approaches at the same time.

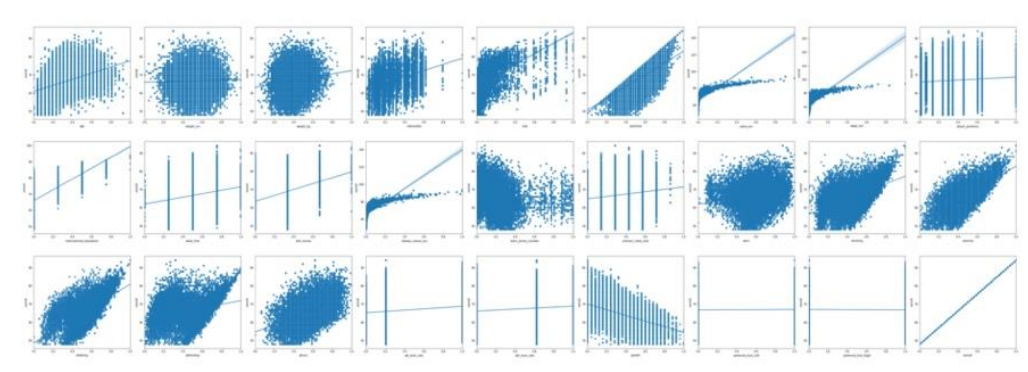

(a)

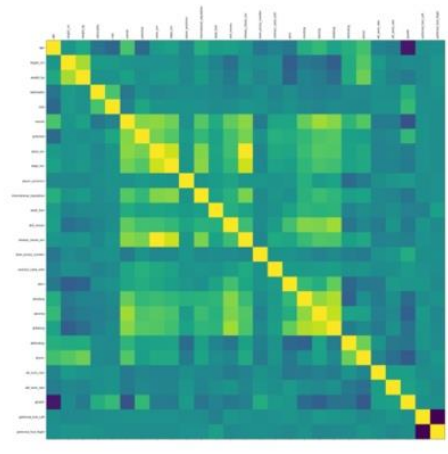

(b)

Figure 1. (a) Scatterplot matrix having the target value "overall" rating of a player and (b) Pearson's correlation coefficient heatmap for the FIFA 20 complete player dataset

\section{VISUALIZATION METHODOLOGY}

\subsection{Design}

The circle wave was proposed by Dave White (Whyte, 2014) as a screen loading animation, programmed in processing. It was adapted to D3.js by Mike Bostock (Bostock, 2019) which was further extended to be used as a medium to visualize correlation in this paper. The original implementation by Bostock has three circle waves with constant amplitude. In this paper, the amplitude of each wave is computed dynamically on the basis of the degree of correlation. Each circle wave is a cosine signal revolving around a pivot, representing a variable. Multiple cosine signals are superimposed, each cosine signal having the same speed and some phase shift, depending on the number of variables to be correlated. The amplitude of each wave gives an idea about the Pearson's correlation coefficient between the variable it represents and the target variable. The amplitude is inversely proportional to the correlation coefficient. This means that higher the correlation of a field, the more its circle wave approximates an exact circle. Circle waves of different colours are used to represent different variables. The attributes having the highest correlation with the target attribute are also ranked in the legend of the visualization. 


\subsection{Exploring a Dataset}

The proposed visualization technique is applied to explore the FIFA 20 complete player dataset (Leone, 2019). This dataset is primarily selected because it contains a comprehensive list of all currently active football players. Secondly, the dataset has a wide set of features which can be used for analysis and has almost negligible missing data. This dataset is present in a tabular form where the columns are various variables and a row represents a player. The dataset has 104 attributes for each of the 18278 players.

It was imperative that a few fields are filtered out from the dataset in order to narrow down the scope of the visualization. Majority of the fields were ratings for granular attributes like "sprint speed", "shot power" and "long pass". These fields were removed from the dataset since they had parent attributes with aggregated scores i.e. "pace", "shooting", "passing", "dribbling", "defending" and "physicality" present in the dataset. Using these parent attributes paints a better picture about the correlation between the players' intrinsic (pace, passing, skill level, etc.) and extrinsic (wage, market value, etc.) attributes. The visualization focuses only on the outfield players, leading to the omission of attributes related to goalkeeping like "diving" and "reflexes" from the dataset. A Pearson's correlation coefficient matrix is generated once the dataset has been cleaned up and all categorical data has been encoded.

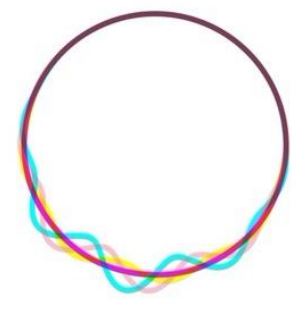

(a)

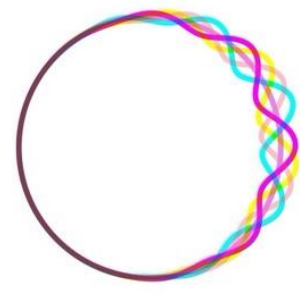

(b)

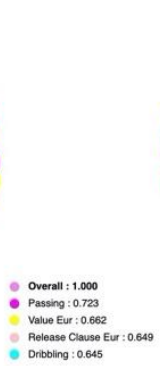

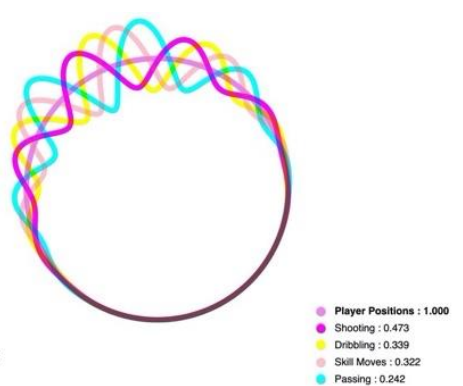

(c)

Figure 2. Using circle waves to show variables highly correlated with the target variables. The target variable is "Value Eur" in (a), "Overall" rating of a player in (b) and "Player Positions" in (c)

Figure 2 shows how circle waves can be utilized to view variables highly correlated with the target variable. Consider figure 2(a), the target value is "value eur", and the highest correlated variables are "release clause eur", "wage eur", "club" and "overall". This makes sense as a player's value, logically, should be highly correlated with their wages, the club they play for and their release clause. The variable "release clause eur" has the highest correlation coefficient, 0.993 and the circle wave associated with it is hardly differentiable from the circumference itself. In contrast, consider figure 2(c), the target variable is "player positions" and the highest correlated variable is "shooting" having a correlation coefficient of 0.473 . The circle wave associated is far away from the circumference. The circle wave associated with the field "passing" is further away since it has a correlation coefficient of 0.242 .

\section{CONCLUSION}

This paper introduces a novel visualization technique to examine multivariate correlation and demonstrates its application on the FIFA 20 complete player dataset. To the best of my knowledge, idioms used to visualize correlation have been limited to solar correlation maps, scatterplots, heatmaps and their variations. Using circle waves to visualize correlation tasks is the novel concept presented in this paper.

The viewer can intuitively understand the magnitude of correlation between different attributes since it utilizes their subconscious perception of visual harmony. Highly correlated attributes will have a neat and orderly visualization whereas attributes having low correlation will lead to a chaotic visualization. This visualization provides a clean depiction of the correlation task and does not overload the viewer with a profusion of information. However, the visualization is restricted to correlating a limited number of attributes for it to remain elegant. Additionally, despite being a novel concept, the visualization can solely be used for 
very specific tasks where the analysis requires multiple target variables to be investigated and a high-level intuition about the correlation magnitude is enough.

This paper lays the groundwork for applying circle waves to visualize correlation tasks. The future work includes testing the technique with different datasets and evaluating its efficacy with a user study. Furthermore, this visualization idiom can be extended to include multiple encoding channels. For example, wave specific features like the stroke width, speed, phase shift and radius of movement can also be utilized to encode information in the visualization. The visualization can be extended to the third dimension, thus achieving another level of information encoding. Extending this visualization to be projected in an augmented reality environment seems achievable as well. With the advancements in technology, applications of the proposed visualization technique's potential know no bounds.

\section{REFERENCES}

Blum, A.L. and Langley, P. (1997). Selection of relevant features and examples in machine learning. Artificial Intelligence, [online] 97(1-2), pp.245-271. Available at: https://www.science direct.com/science/article/pii/S0004370297000635.

Bostock, M. (2019). Circle Wave. [online] observablehq.com. Available at: https://observablehq.com/@mbostock/circlewave.

Cawthon, N. and Moere, A.V. (2007). The Effect of Aesthetic on the Usability of Data Visualization. 2007 11th International Conference Information Visualization (IV '07).

Friendly, M. (2002). Corrgrams. The American Statistician, 56(4), pp.316-324.

Horvath, S. and Dong, J. (2008). Geometric Interpretation of Gene Coexpression Network Analysis. PLoS Computational Biology, 4(8), p.e1000117.

Leone, S. (2019). FIFA 20 complete player dataset. [online] kaggle.com. Available at: https://www.kaggle.com/stefanoleone992/fifa-20-complete-player-dataset\#players_20.csv.

Rensink, R.A. and Baldridge, G. (2010). The Perception of Correlation in Scatterplots. Computer Graphics Forum, 29(3), pp.1203-1210.

Robertson, G., et al. (2008). Effectiveness of Animation in Trend Visualization. IEEE Transactions on Visualization and Computer Graphics, 14(6), pp.1325-1332.

Whyte, D. (2014). Circle wave II. [online] dribbble.com. Available at: https://dribbble.com/shots/1698964-Circle-waveII.

Wilkinson, L. and Friendly, M. (2009). The History of the Cluster Heat Map. The American Statistician, 63(2), pp.179-184.

Wong, B. (2010). Color coding. Nature Methods, [online] 7(8), pp.573-573. Available at: https://www.nature.com/articles/nmeth0810-573.

Zapf, S. and Kraushaar, C. (2017). A new visualization to beautifully explore correlations. [online] O'Reilly Media. Available at: https://www.oreilly.com/content/a-new-visualization-to-beautifully-explore-correlations [Accessed 26 May 2020]. 